\title{
Wissenschaftliche Karriere und Beschäftigungsbedingungen
}

\author{
Organisationssoziologische Überlegungen zu den Grenzen neuer \\ Steuerungsmodelle an deutschen Hochschulen
}

\author{
Von Otto Hüther und Georg Krücken
}

\begin{abstract}
Zusammenfassung: Die Karriere- und Beschäftigungsbedingungen an Universitäten sind ein für die Soziologie wichtiger Forschungsgegenstand mit darüber hinausgehenden hochschulpolitischen Implikationen. In der Diskussion fehlt jedoch ein organisationssoziologischer Zugang, der die Besonderheiten der Universitätsorganisation in den Vordergrund rückt. Anknüpfend an Luhmanns Unterscheidung zwischen Organisations- und Personalmacht wird deutlich, dass deutschen Universitäten die klassischen, in anderen Organisationen vorhandenen Machtressourcen in zentralen Fällen gar nicht zur Verfügung stehen: Weder kann die Organisation über die Mitgliedschaft so disponieren, wie dies in Unternehmen der Fall ist („Organisationsmacht“), noch stehen der Organisation die in der öffentlichen Verwaltung vorhandenen Aufstiegs- und Karriereanreize zur Verfügung („Personalmacht"). Wir sehen hierin eine in der soziologischen und hochschulpolitischen Diskussion häufig übersehene Grenze der Einsatzes neuer Steuerungsmodelle im Hochschulbereich. Diese für deutsche Universitäten spezifische Grenze wird in dem Beitrag sowohl mit Bezug auf die Organisationssoziologie und „New Public Management“-Forschungen als auch im Vergleich mit anderen Ländern herausgearbeitet.
\end{abstract}

\section{Einleitung}

Die Art der wissenschaftlichen Karriere und die Beschäftigungsbedingungen der Professoren sowie des wissenschaftlichen Nachwuchses sind ein traditioneller Forschungsgegenstand in der Universitätsforschung. Eine erste empirische Studie zum wissenschaftlichen Nachwuchs in Deutschland wurde so schon 1908 von Franz Eulenburg vorgelegt, in der die Lage der Privatdozenten und Extraordinarien untersucht wurde. Bereits in dieser Studie findet sich ein Themenkomplex, der auch in neueren Studien zu diesem Thema zentral ist: die Frage nach der Unsicherheit und dem Risiko einer wissenschaftlichen Karriere (Eulenburg 1908). Eine weitere klassische Behandlung des Karrieresystems findet sich dann bei Max Weber, der 1919 in seinem Vortrag „Wissenschaft als Beruf“ ebenfalls mit dem Begriff des „Hasard“ das hohe Risiko der wissenschaftlichen Karriere in Deutschland betont. Weber zeigt in seiner Abhandlung durch einen Vergleich mit dem amerikanischen Karrieresystem, dass die Unsicherheit der akademischen Laufbahn in Deutschland besonders hoch ist, weil erst mit dem späten Erreichen der ersten Professur eine unbefristete Position erreicht wird. Ein weiterer Aspekt, der sich bereits bei Weber findet, ist die spezifische intrinsische Motivlage von Wissenschaftlern. Wissenschaft als Beruf, so Weber, benötigt „Leidenschaft“, „innere Hingabe“ und die reine Orientierung an der Sache (Weber 1922).

In der Tradition beider Autoren bewegen sich dann auch neuere Untersuchungen zu wissenschaftlichen Karrieren, die primär vom einzelnen Wissenschaftler ausgehen und die soziale Lage und / oder die Motivation untersuchen (z.B. Joas / Bochow 1987; Enders 1996; Klecha / Krumbein 2008). International vergleichende Arbeiten liegen insbesondere aus zwei Perspektiven vor. Diese basieren zum einen auf Befragungen von Professoren und wissenschaftlichen Mitarbeitern zu Arbeits- und Beschäftigungsbedingungen (z.B. Boyer et al. 1994; European Review 2010), zum anderen in Form von beschreibenden Länderberichten (z.B. Enders 2000 a; Kreckel 2008 a). Im internationalen Vergleich zeigt sich dann, dass im Hinblick auf das Risiko und die herausgehobene Position der Professoren in Bezug auf die Beschäftigungs- 
sicherheit, wie sie schon von Eulenberg und Weber beschrieben wurden, im deutschen Hochschulsystem kaum Veränderungen stattgefunden haben. Karrieresysteme und Beschäftigungsbedingungen waren und sind ein zentrales Unterscheidungskriterium nationaler Hochschulsysteme (Musselin 2010).

Insgesamt zeigt sich, dass die bisherige nationale und international vergleichende Forschung einen deutlichen Schwerpunkt auf die Auswirkungen von Karriere- und Beschäftigungsbedingungen auf die individuelle Ebene der Wissenschaftler legt. Auffällig ist jedoch, dass die Organisationsebene zumeist ausgeblendet bzw. allenfalls gestreift wird. Im Gegensatz dazu nimmt unser Beitrag die Organisation als Ausgangspunkt zur Betrachtung der Karrieremuster und Beschäftigungsbedingungen insbesondere im deutschen System. Der Beitrag betrachtet die organisationalen Bedingungen und Auswirkungen unterschiedlicher Karriere- und Beschäftigungsstrukturen. Aus dieser Perspektive treten dann auch weniger die Fragen nach Sicherheit / Unsicherheit oder Motivlage der Wissenschaftlerinnen und Wissenschaftler in den Vordergrund, sondern organisationale Machtstrukturen und die Frage nach der organisationalen Kontrolle und Steuerung ihrer Mitglieder. ${ }^{1}$

Damit rücken auch ganz grundsätzliche Aspekte der institutionellen Bedingungen der Hierarchie als Governancemechanismus in Organisationen und anderen gesellschaftlichen Handlungs- und Entscheidungskontexten in den Blickpunkt. Während nämlich in der allgemeinen soziologischen Governanceforschung zuweilen eine Gleichsetzung von Hierarchie und Organisation zu finden ist (z.B. Wiesenthal 2000), weist die Organisationstheorie gerade darauf hin, dass Hierarchie in Organisationen eine variable Größe ist, die, wie zum Beispiel im Falle von Professionsorganisationen (Mintzberg 1983), nicht einfach vorausgesetzt werden kann. Dies weist darauf hin, dass Hierarchie zwingend auf institutionelle Absicherungsmechanismen angewiesen ist (z.B. Scharpf 1997), deren Vorhandensein geprüft werden muss. ${ }^{2}$ Ein zentraler institutioneller Absicherungsmechanismus von Hierarchie sind dabei Macht- und Sanktionspotentiale. Unser Beitrag prüft deshalb auch, ob diese institutionelle Voraussetzung von Hierarchie aufgrund der Karriere- und Beschäftigungsbedingungen an deutschen Universitäten überhaupt vorhanden ist. Eine solche Prüfung legt die Nutzung eines instrumentell ausgerichteten Machtkonzeptes innerhalb der Organisation nahe, während stärker diskursiv ausgerichtete Machtkonzepte, wie sie etwa in Clegg et al. (2009: 290ff.) diskutiert werden, für eine solche Überprüfung weniger geeignet erscheinen. Einen solchen instrumentellen Zugriff auf Macht- und Sanktionspotentiale bietet die Luhmannsche Unterscheidung von Organisations-

1 Veröffentlichungen, die sich mit dem geringen Frauenanteil insbesondere auf der Ebene der Professorenschaft auseinandersetzen, betonen neben individuellen Karrieremustern und Motivlagen ebenfalls Machtaspekte (z.B. Krais 2000; Wissenschaftsrat 2007). Jedoch wird Macht hier primär in gesellschaftlich institutionalisierten Ungleichheitsmustern und im Zugang zu sozialen und meist informell strukturierten Netzwerken gesehen und weniger in der Formalstruktur der Organisation und ihren Machtressourcen. Vgl. hierzu auch die Sekundäranalyse von Gross / Jungbauer-Gans (2007), die vor allem auf die Bedeutung des Zugangs zu diesen Netzwerken zur Erklärung unterschiedlicher Karrierechancen aufmerksam macht.

2 Dieses Prüfungserfordernis gilt grundsätzlich auch für die Makroebene des Gesellschaftssystems, wo es in der Governanceliteratur häufig zu einer Gleichsetzung von Staat und Hierarchie kommt. Auch wenn aufgrund der Geltung des formalen Rechts und seiner Durchsetzung durch das Rechtssystem hier erhebliche Machtpotentiale des Staates anzunehmen sind, weist die soziologische Planungs- und Steuerungsdebatte bereits seit der Implementationsforschung der späten 1970er Jahren auf die Grenzen staatlich-hierarchischer Entscheidungen hin, die auch auf den Mangel an institutioneller Absicherung bzw. Durchsetzbarkeit zurückzuführen sind. 
und Personalmacht (Luhmann 1975: 104ff.), ${ }^{3}$ die in diesem Beitrag zugrunde gelegt wird. Luhmann zufolge verfügen Organisationen über Organisations- und / oder Personalmacht. Diese Machtressourcen variieren jedoch je nach Organisationstypus. Unternehmen verfügen über Organisationsmacht, da sie über die Fortdauer der Mitgliedschaft disponieren können. Die öffentliche Verwaltung kann bei ihren zumeist verbeamteten Mitgliedern hingegen nicht über die Zugehörigkeit zum Organisationssystem disponieren. Allerdings verfügt sie über Personalmacht, d.h. die Möglichkeit, die Karrierewege ihrer Mitglieder zu beeinflussen. Vor diesem Hintergrund soll unsere organisationssoziologische Analyse zeigen, dass Universitäten im Hinblick auf ihre wissenschaftlichen Mitglieder weder über Organisationsmacht noch über Personalmacht verfügen. Trotz weit reichender Angleichungen der Organisation „Universität“ an Organisationen anderer gesellschaftlicher Bereiche bilden, so unsere These, die Karrieremuster und Beschäftigungsbedingungen an deutschen Universitäten eine fundamentale Barriere für die Angleichung der Organisation „Universität“ an andere Organisationstypen. Der Grund hierfür ist, dass die institutionellen Voraussetzungen der Hierarchie in der Organisation eingeschränkt sind und deshalb Hierarchie als Koordinationsmechanismus kaum einsetzbar ist.

Diese These wird in zwei Schritten entwickelt. Im ersten Schritt (Teil 2) werden wir mit Bezug auf international vorzufindende Universitätsreformen, die in den Rahmen eines allgemeinen Diskurses um „New Public Management“ eingebettet sind, herausarbeiten, dass die strategische Handlungsfähigkeit der Organisation „Universität“ immer stärker in den Blick rückt. Das bedeutet einen grundlegenden Wandel, der auch die traditionelle Organisationssoziologie der Universität, die wir zu Beginn von Teil 2 als Kontrastfolie kurz skizzieren, in Frage stellt. Im Rahmen dieser Reformen findet eine Öffnung gegenüber den in anderen Organisationskontexten vorherrschenden Strukturmustern und Instrumenten statt. Die im ersten Schritt geführte Diskussion hebt wichtige Veränderungsprozesse hervor. Ihre Schwäche besteht jedoch darin, die institutionellen Voraussetzungen der hierarchischen Steuerung, die als grundlegender Bestandteil dieses Wandels angesehen wird, in derartigen Veränderungsprozessen auszublenden. Folglich kommt es zu einer Überbetonung von Angleichungsprozessen zwischen Organisationen. Diese Schwäche versuchen wir im zweiten Schritt (Teil 3) im Rekurs auf das Luhmannsche Machtkonzept zu kompensieren. Dabei wird gezeigt, dass sich der Unterschied zwischen Universitäten und anderen Organisationen nicht auflöst, weil der Zugriff der Organisation auf ihre wissenschaftlichen Mitglieder gerade an deutschen Universitäten nach wie vor sehr begrenzt ist. Dies gilt sowohl für Professoren, die ihre Karriere typischerweise außerhalb der Organisation verfolgen, als auch für den wissenschaftlichen Nachwuchs, auf dessen Karrieremöglichkeiten die Organisation ebenfalls kaum Einfluss nehmen kann. Im abschließenden Teil 4 fassen wir die Ergebnisse zusammen. Dabei wird von uns auch der neuere Versuch, mit Hilfe monetärer Anreizsteuerung den Mangel an Organisations- und Personalmacht zu kompensieren, einer Einschätzung und Bewertung unterzogen.

\section{Universitäten als vollständige Organisationen mit Akteursstatus}

Traditionell wurden Universitäten als spezifische Organisationen angesehen, die sich fundamental sowohl von Wirtschafts- als auch Verwaltungsorganisationen unterscheiden. Verwiesen wird in diesem Zusammenhang meist auf drei Perspektiven der Organisationsforschung: Universitäten sind lose gekoppelte Systeme (Weick 1976), organisierte Anarchien (Cohen et

3 Interessanterweise taucht diese Unterscheidung in Luhmanns organisationssoziologischem Haupt- und Spätwerk „Organisation und Entscheidung“ nicht mehr explizit auf, wie ohnehin Machtfragen hier nur von untergeordneter Bedeutung sind und auf wenigen Seiten abgehandelt werden (Luhmann 2000: 200ff.). Jedoch finden sich Überlegungen zur Organisations- und Personalmacht an anderer Stelle, sei es in den Ausführungen zur Kontingenz der Mitgliedschaft in Organisationen (Luhmann 2000: 80ff.), sei es in den Überlegungen zu Karrieren in Organisationen (Luhmann 2000: 101ff.; 297ff.). 
al. 1972) und der Prototyp der professionellen Bürokratie (Mintzberg 1983). Alle drei genannten Perspektiven machen auf Eigenarten der Organisationsform Universität im Vergleich zum Idealtypus der rationalen bzw. bürokratischen Organisation aufmerksam.

Die Perspektive der losen Kopplung betont das Fehlen von engen Verbindungen zwischen Organisationsteilen und daraus folgend die Problematik einer gesamtorganisatorischen Steuerung. Die Professionsperspektive fokussiert insbesondere die Allokation von Machtpotentialen im operativen Kern von Organisationen, der in Universitäten aus den Wissenschaftlern besteht, den Einbau von Kollegialitätsprinzipien und den latenten Konflikten zwischen der Logik der Profession und der Logik der Hierarchie. Beide Organisationsperspektiven markieren damit eine vergleichsweise schwache Stellung der Organisationsführung gegenüber dem operativen Kern. Das Bild der organisierten Anarchie hingegen stellt Entscheidungsprozesse in Universitäten in den Mittelpunkt und betont, dass unter der Bedingung von problematischen Präferenzen, unklaren Technologien und fluktuierender Partizipation zweckrationale Entscheidungen nicht die Regel darstellen.

Während in der Forschung zu Universitäten traditionell deren Sonderstatus als Organisation hervorgehoben wurde, wird dies durch die international feststellbaren Reformen der Universitäten seit Anfang der 1980er Jahre zumindest in Frage gestellt. In England beginnt ab den 1980er Jahren eine Entwicklung, die traditionelle Regelungsstrukturen der europäischen Universitäten umgestaltet (z.B. Leisyte et al. 2006; Risser 2003). Auch die Niederlande werden relativ früh von dieser Entwicklung erfasst (z.B. de Boer et al. 2006). Ab den 1990er Jahren ist dann in fast allen westlichen Hochschulsystemen eine Veränderung der traditionellen Governancestrukturen zu bemerken. Es bildet sich ein ,global model“ (Baker / Lenhardt 2008: 1) der Hochschulgovernance heraus, das nicht nur in Europa, sondern weltweit den Diskurs um Hochschulreformen dominiert. Reformmaßnahmen in den nationalen Universitätssystemen können deshalb nur noch dann Legitimität beanspruchen, wenn sie sich im Rahmen dieses „policy paradigm“ (Hall 1993) bewegen (Lange / Schimank 2007: 525).

Geprägt ist dieses globale Modell durch eine Verstärkung der Konkurrenz zwischen den und innerhalb der Universitäten, eine Stärkung der internen Hierarchie, eine Schwächung des Kollegialitätsprinzips und eine verstärkte Außensteuerung gegenüber einer Detailsteuerung durch den Staat (z.B. de Boer et al. 2007 a; Kehm / Lanzendorf 2006). Bezeichnet wird dieses Modell meist als New Public Management (NPM)-Modell. ${ }^{4}$

Sowohl die Bezeichnung als auch die mit dem Modell verbundenen Instrumente verweisen dabei auf das allgemeine NPM-Konzept. Dieses zielte nicht originär auf einen universitären Anwendungskontext, sondern es wurde entwickelt, um allgemein staatliche Verwaltungseinrichtungen zu reformieren und die Aufgaben des Staates möglichst effektiv und effizient zu erfüllen. Im Mittelpunkt der binnenstrukturellen Dimension ${ }^{5}$ steht dabei die innere Struktur der Verwaltungsorganisation, die in Bezug auf die Entscheidungsstrukturen, die Personalpolitik, die Leistungsmessung und die Steuerung an privatwirtschaftliche Unternehmen angeglichen werden soll (allgemein zum NPM-Konzept z.B. Bogumil et al. 2007; Vogel 2006; für die internationalen Entwicklungen z.B. Pollitt et al. 2007).

Die Anwendung des NPM-Konzeptes auf Verwaltungen und Universitäten geht dann aber davon aus, dass diese mit privatwirtschaftlichen Dienstleistungsunternehmen vergleichbar

4 Zuweilen findet sich auch die Begriffe „New Managerialism“ oder „Entrepreneurial Universities“.

5 Im Gegensatz dazu steht die ordnungspolitische Dimension, die dadurch geprägt ist, dass für den gesamten öffentlichen Sektor geprüft wird, ob bestimmte Aufgaben durch den Staat oder aber durch private Anbieter gelöst werden können. Es geht hier also um die Begrenzung staatlichen Handelns auf Kernaufgaben, wenn die Umsetzung des NPM - wie in England, jedoch weniger in Deutschland primär über Privatisierungsmaßnahmen erfolgt. 
sind. Während also die oben beschriebenen Organisationsperspektiven die Besonderheiten von Universitäten betonen, wird durch die international vorzufindenden Reformen im Rahmen des NPM-Konzeptes eine deutliche Angleichung angestrebt bzw. für möglich gehalten.

Dass sich Organisationen angleichen, ist im Rahmen der soziologischen Organisationstheorie kein neuer Gedanke. Hier ist zum Beispiel auf die Isomorphismusthese des Neoinstitutionalismus zu verweisen (DiMaggio / Powell 1983). Auch in der historischen Entwicklung der Universitäten sind Angleichungstendenzen zwischen Universitäten kein Sonderfall. Man denke nur an die weltweite Durchsetzung des humboldtschen Universitätsmodells im 19. Jahrhundert (Altbach 1991). Das wirklich Neue an den momentan vorzufindenden Entwicklungen scheint dabei zu sein, dass als Anpassungsvorbild nicht Organisationen gleichen Typs verwendet werden und der Isomorphismus auch nicht seinen Ursprung im organisationalen Feld der Universitäten hat (Krücken / Meier 2006). Die Angleichung ist vielmehr deutlich basaler angelegt, weil als Vorbild ein allgemeines Modell der Organisation verwendet wird, wie es sich insbesondere im Bereich der Wirtschaft finden lässt. Besonders deutlich wird dies in einem zentralen Aufsatz aus dem Jahr 2000 von Nils Brunsson und Kerstin Sahlin-Andersson. Die Autoren argumentieren hier, dass die Reformen im Zuge des NPM als ein Versuch interpretiert werden können, öffentliche Verwaltungen und Universitäten als „,complete organizations “ mit Akteursstatus zu konstruieren. „Complete organizations“ zeichnen sich ihnen zufolge durch Gesamtidentität (Autonomie, kollektive Ressourcen, Abgrenzung zur Umwelt, Selbstwahrnehmung als Organisation), Hierarchie (Koordination und Kontrolle, internes Management) und Rationalität (eindeutige Ziele, Messung und Zurechnung von Leistungen) aus.

Im Vergleich zu den traditionellen (Organisations-)Beschreibungen der Universitäten wird anhand dieser Kriterien deutlich, dass Universitäten tatsächlich ,incomplete organizations“ sind: Universitäten sind lose gekoppelte Systeme ohne Gesamtidentität, Hierarchie widerspricht dem in der Professionsperspektive betonten Kollegialitätsprinzip, und Rationalität im Sinne von Brunsson / Sahlin-Andersson (2000) ist nicht mit der Beschreibung organisierter Anarchien, deren Prozesse typischerweise nicht zweckrational verlaufen, in Einklang zu bringen.

Auch wenn Brunsson / Sahlin-Andersson (2000: 730 f.) betonen, dass es sich bei den beschriebenen Konzepten zunächst um Konstruktionen und Diskurse handelt und es abzuwarten ist, ob bzw. wie sich die Reformen des öffentlichen Sektors und der Universitäten tatsächlich auswirken, finden sich ohne Zweifel sowohl im Rahmen der internationalen (z.B. de Boer et al. 2007 b; Krücken / Meier 2006) als auch der deutschen (Meier 2009; Hüther 2010) Reformen Hinweise für diese Entwicklung. Im Folgenden werden wir die drei von Brunsson / SahlinAndersson (2000) als zentral behandelten Kriterien zur Identifizierung von „complete organizations“ - Gesamtidentität, Hierarchie, Rationalität - als Heuristik behandeln und für den deutschen Fall beispielhaft erläutern.

\section{Gesamtidentität}

Eine Reihe von Maßnahmen der jüngeren Universitätsreformen stärkt die Bildung einer Gesamtidentität der individuellen Universitäten. Die Autonomie der Organisation steigt z.B. dadurch, dass sich im NPM-Modell der Staat aus der bürokratischen Detailsteuerung zurückzieht. Stattdessen soll lediglich eine Außensteuerung im Sinne eines „framework steering“ (van Vught 1997: 132) oder eines ,steering at a distance“ (Braun / Merrien 1999 a: 17) vorgenommen werden. Im deutschen Universitätssystem, in dem der Staat traditionell einen besonders großen Einfluss hatte, wird an dieser Stelle mit einer jahrhundertealten Tradition gebrochen. Das neue Instrument von Ziel- und Leistungsvereinbarungen zwischen Hochschule und Staat - und damit der Versuch einer Umstellung der Input- zu einer Outputsteuerung - ist diesem Bereich zuzuordnen, da hier die Hochschulorganisation als Einheit adressiert wird. 
Auch der Aufbau von kollektiven Ressourcen kann nachvollzogen werden. In Deutschland wurde durch die Einführung von Globalbudgets, die der Universität insgesamt zur Verfügung gestellt werden, genau dieser Effekt erfüllt. Während in der kameralistischen Haushaltsführung der Etat aufgrund von individualisierten Aufstellungen zugeteilt wurde, wird nun also eine gemeinsame kollektive Ressourcenausstattung gewährt, die von der Organisation verteilt wird.

In Bezug auf die Selbstwahrnehmung als Organisation kann gleichfalls festgestellt werden, dass diese durch die neueren Reformen deutlich erhöht wird. Ein zentraler Aspekt ist dabei der Aufbau von Wettbewerb zwischen Universitäten. In Deutschland findet dieser zum einen auf Länderebene, zum anderen aber auch auf der Bundesebene - Stichwort „Exzellenzinitiative“ - statt. Gleichfalls findet sich eine Zunahme der allgemeinen Rechenschaftspflicht der Organisation. So werden Universitäten durch formale Qualitätskontrollen, Hochschulrankings und -evaluationen sowie Zielvereinbarungen zunehmend als Gesamtorganisation adressiert.

Die neueren Reformen weisen in der Tendenz demnach tatsächlich darauf hin, dass das Merkmal der Gesamtidentität der Organisation gestärkt wird und somit eine Entwicklung in Richtung ,complete organization“ vonstatten geht.

\section{Hierarchie}

Die Stärkung der internen hierarchischen Beziehungen vollzieht sich durch eine Verlagerung von Entscheidungskompetenzen staatlicher Akteure sowie insbesondere der akademischen Selbstverwaltungseinheiten in Richtung der Hochschulleitungen und Dekane. Die Literatur geht hier insgesamt davon aus, dass es zu einer Hierarchisierung der Universitäten kommt, in deren Verlauf das zuvor dominierende Prinzip der akademischen Selbstverwaltung an Bedeutung verliert (für einen aktuellen Überblick Hüther 2010).

Gleichzeitig sind Tendenzen offensichtlich, das Hochschulmanagement stärker zu professionalisieren (Blümel et al. 2010; Krücken et al. 2010). Dies ist sowohl für die Managementspitze, z.B. durch verlängerte Amtszeiten, als auch für den gesamten Verwaltungsapparat der Universitäten zu bemerken. Letzteres wird deutlich durch die immer weitere Differenzierung und Spezialisierung der Hochschulverwaltung, die damit einhergeht, dass die Universitäten ihre organisatorische Zuständigkeit in immer mehr Handlungsbereiche ausdehnen - vom Technologietransfer über die Frauengleichstellung bis zur Personalentwicklung. Hiermit ist auch ein deutlich verändertes Selbstverständnis auf Seiten der Verwaltung sowie ihrer Mitarbeiterinnen und Mitarbeiter verbunden, da das Leitbild des eigenständig-entscheidungsorientierten Handelns die klassische bürokratische Rationalität zunehmend überformt. Dieser Prozess der Professionalisierung und Differenzierung der Verwaltung lässt sich sowohl national (Blümel et al. 2010; Krücken et al. 2010) als auch international (Whitchurch 2006; Rhoades / Sporn 2002; Gornitzka et al. 1998) nachweisen.

Vor allem die Stärkung der Hochschulleitungen und Dekane, die zunehmend weniger als ,primus inter pares“ innerhalb des professoralen Kollegiums und verstärkt als entscheidungsbefugte Spitze einer Organisation bzw. Organisationseinheit gesehen werden, aber auch die Professionalisierungs- und Spezialisierungstendenzen innerhalb der Verwaltung, die die wis- 
senschaftlichen und nicht-wissenschaftlichen Mitarbeiter an den Zielen der Gesamtorganisation auszurichten versuchen, weisen deutlich in Richtung einer verstärkten Hierarchie.

\section{Rationalität}

Eine verstärkte Rationalität lässt sich z.B. aus den Profilbildungsprozessen der einzelnen Universitäten ableiten, die hierdurch zumindest ihre Zielsetzungen konkretisieren (Meier / Schimank 2002). Gleichfalls finden sich vermehrt Leitbilder, die die Mission der Universitäten festlegen sollen. In ihrer noch unveröffentlichten Analyse zu Hochschulleitbildern in Deutschland stellt Kosmützky (2010) heraus, dass heutzutage nahezu alle Hochschulen über ein Leitbild verfügen, das ganz unterschiedliche Funktionen nach innen und außen erfüllt. Auch wenn diese Leitbilder von Skeptikern lediglich als für die Organisation folgenloses ,window-dressing" angesehen werden, so ist darin doch der Versuch zu sehen, Ziele der Organisation insgesamt $\mathrm{zu}$ benennen. Es finden demnach gegenwärtig individuelle Profilbildungsprozesse statt, die - in engem Zusammenhang mit den beiden zuvor genannten Punkten der Gesamtidentität und Hierarchie - die Universität als Organisation schärfer konturieren.

Hinzu kommt die vielfältige Ausweitung von Leistungsmessung und Evaluation, die letztendlich davon ausgeht, dass die betrachteten Einheiten auch verantwortlich für die gemessenen Resultate sind. $\mathrm{Zu}$ denken wäre hier an die in vielen Landeshochschulgesetzen geforderte Evaluation von Forschung und Lehre. Damit einher geht die Zurechenbarkeit von Fehlleistungen sowohl auf die Gesamtorganisation als auch auf ihre Teile. Wird auf der Landesebene verblieben, dann ist auch der in den meisten Bundesländern abgeschlossene Aufbau der indikatorengestützen Mittelvergabe zu betrachten. Gemessen wird hier meist die Leistung in der Lehre, Forschung, Gleichstellung und Internationalisierung, wobei zum Beispiel die Anzahl der Absolventen in der Regelstudienzeit, die eingeworbenen Drittmittel oder die Anzahl der Promotionen als Indikatoren benutzt werden (Hartwig 2006; Jaeger 2006).

Die Ausführungen zeigen, dass die Tendenzen hin zur Entwicklung von Universitäten als „complete organizations“ (Brunsson / Sahlin-Andersson 2000) kaum zu übersehen sind. Insofern gibt es auch deutliche Angleichungstendenzen zwischen Wirtschaftsorganisationen, Verwaltungen und Universitäten.

Dennoch ist bereits jetzt und vor unserer Diskussion der zur Umsetzung fehlenden Personalund Organisationsmacht darauf hinzuweisen, dass diese Tendenzen nicht im Sinne eines umfassenden gleichgerichteten globalen Modells überschätzt werden sollten. Dafür spricht erstens, dass vergleichende Studien zu den Reformen an Universitäten in verschiedenen Ländern darauf hinweisen, dass nationale Traditionen durchaus auch weiterhin eine Rolle spielen (vgl. z.B. die Studien in Krücken et al. 2007; Braun / Merrien 1999 b; Kehm / Lanzendorf 2006), und zum Teil auch ineffektive Strukturen im Verlauf der Reformen eingeführt wurden (für Frankreich Mignot 2003, für Italien Capano 2008). Auch wenn NPM-Reformen im Hochschulbereich transnationalen Charakter haben, werden diese von den einzelnen Staaten unterschiedlich adaptiert bzw. es findet ein differenzierter „translating-“ und „editing-“ Prozess (Sahlin-Andersson 2002) statt.

Zweitens werden innerhalb der einzelnen Länder gleichzeitig sich zum Teil widersprechende „narratives of public services reform“ (Ferlie et al. 2008: 334) genutzt. So überlagern sich Vorstellungen von NPM, Netzwerksteuerung und Neo-Weberianismus, die sich mehr oder weniger auch in den Hochschulreformen der einzelnen Hochschulsysteme wiederfinden lassen (Ferlie et al. 2008).

Drittens ist für den deutschen Fall darauf hinzuweisen, dass zwar die Reformtendenzen in Richtung Angleichung gehen, zwischen den Bundesländern aber erhebliche Unterschiede vor- 
handen sind. Diese Unterschiede betreffen z.B. die Kompetenzausstattung oder die Wahl- und Abwahlregeln des Hochschulrates oder der Hochschulleitung (z.B. Hüther 2009; Lange 2009; Kehm / Lanzendorf 2006). Insgesamt zeigt sich dann auch, dass in den deutschen Landeshochschulgesetzen sich zum Teil widersprechende Vorstellungen zur Reform der Universitäten finden lassen (Hüther 2010), wie dies auch von Ferlie et. al. (2008) für nationale Systeme gezeigt wurde.

Neben diesen Einschränkungen im Hinblick auf die nationale und regionale Umsetzung des NPM-Modells und damit der Angleichung der Universitäten lässt sich zudem erwarten, dass einige Besonderheiten des Organisationstyps erhalten bleiben. So ist aus organisationssoziologischer Perspektive zu vermuten, dass Charakteristika loser Kopplung, diskursive Verfahren und die Orientierung am Kollegialitätsprinzip sowie die Probleme der Zielbestimmung und Leistungsmessung weiterhin von hoher Relevanz sind und auch in Zukunft bleiben werden.

Mit Hilfe der einleitend benannten Unterscheidung von Organisations- und Personalmacht als den zentralen organisationalen Machtressourcen möchten wir in Teil 3 jedoch eine in der Forschung bislang nicht thematisierte Grenze der in Teil 2 beschriebenen Tendenzen, insbesondere in Bezug auf die Stärkung der internen Hierarchie, diskutieren. Diese sehen wir in den Karriere- und Beschäftigungsbedingungen an deutschen Universitäten, die anders als in anderen Ländern nur geringe Möglichkeiten des Einsatzes der beiden Machtressourcen erlauben.

\section{Karriere- und Beschäftigungsbedingungen als Barrieren der Akteurs- werdung der Universitäten}

Während das zuvor dargestellte Konzept der „complete organization“ von einer stärkeren Hierarchie aufgrund von Verlagerungen von Entscheidungskompetenzen innerhalb der Organisation ausgeht, wird nicht berücksichtigt, dass Hierarchie auf institutionelle Absicherungen angewiesen ist, die nicht durch eine reine Kompetenzverlagerung in Entscheidungssituationen erfasst werden kann. Vielmehr benötigt eine Organisationsleitung mit erhöhten Entscheidungskompetenzen auch Macht- und Sanktionspotentiale gegenüber den Mitgliedern der Organisation, um so die Verbindlichkeit ihrer Entscheidungen abzusichern. Macht- und Sanktionspotentiale innerhalb der Organisation spielen aber im Rahmen der Diskussion um das allgemeine Modell der „complete organization“ keine Rolle und werden generell in der New Public Management-Literatur kaum behandelt. Aus diesem Grund nehmen wir hier eine Ergänzung durch das instrumentelle Machtkonzept von Luhmann mit seiner Unterscheidung von Personal- und Organisationsmacht vor. Bei diesem Vorgehen zeigen sich dann für den deutschen Fall erhebliche Abweichungen nicht nur im Vergleich zu Unternehmen und Verwaltungen, sondern auch im Vergleich zu Universitäten in anderen nationalen Settings. ${ }^{6}$ Daraus ergeben sich dann starke Barrieren in Bezug auf die Konstruktion von ,complete organizations“, wobei die Organisationsschwäche der Universitäten, so unsere These, grundlegend mit den wissenschaftlichen Karrieremustern und Beschäftigungsbedingungen an deutschen Universitäten verknüpft ist, die den Einsatz von Personal- und Organisationsmacht nur in geringem Maße ermöglichen. Hierbei ist zu beachten, dass sowohl die Personal- als auch die Organisationsmacht ihre größte Wirkungskraft weniger in der konkreten Anwendung finden, sondern in der Antizipation der Mitglieder in Bezug auf ihren konkreten Einsatz. Beide Machtquellen sind deshalb dann besonders wirksam, wenn ihr Einsatz als latente Hintergrundmöglichkeit bei der Wahl der Handlungsoptionen durch die Organisationsmitglieder mitläuft. Die eher

6 Diese Abweichungen sind dabei in einem langen historischen Prozess entstanden und haben ihren Ursprung letztendlich in der starken Stellung des einzelnen Professors im deutschen Universitätssystem. Dabei ist die Unabhängigkeit der Professoren in der historischen Entwicklung das zentrale Merkmal für akademische und wissenschaftliche Freiheit, wobei diese Gleichsetzung auch heute noch nachwirkt (vgl. Baker / Lenhardt 2008). 
seltenen Gelegenheiten, in denen die Machtquellen tatsächlich zum Einsatz kommen, also ein Aufstieg oder ein Organisationsausschluss erfolgt, dienen dann auch dazu, zu zeigen, dass die Machtpotentiale vorhanden sind und jederzeit manifest werden können.

\section{Personalmacht}

In typischen Wirtschaftsorganisationen, aber auch Verwaltungen, werden die Handlungen von Mitgliedern unter anderem dadurch gesteuert, dass Vorgesetzte Einfluss darauf haben, ob Mitglieder begehrte Stellen in der Organisation erlangen. Eine Kontrolle von Handlungen wird also dadurch erreicht, dass die Mitglieder begehrte Positionen erreichen wollen und der Erwerb von der Führung abhängt (Luhmann 1975: 106). Über diese „Personalmacht“ verfügen die deutschen Universitäten in der Regel aber nicht.

Zunächst ist in diesem Zusammenhang auf die Karrierewege der deutschen Wissenschaftler hinzuweisen. Hier fällt auf, dass nach dem Erreichen einer Professur keine weiteren Karriereschritte innerhalb des deutschen Wissenschaftssystems vorgesehen sind. Lediglich durch eine Differenzierung der Professuren (C2 bis C4 bzw. W2 bis W3) ergeben sich im deutschen System Unterscheidungen, die primär mit der Steigerung von ökonomischem Kapital verbunden sind, aber für Außenstehende nicht sichtbar gemacht werden. ${ }^{7}$

Aus Sicht der jeweiligen Organisation sind diese Unterscheidungen allerdings machttechnisch nicht nutzbar, da ein interner Aufstieg der Professoren aufgrund des Hausberufungsverbots $^{8}$ in der Regel nicht möglich ist. Karrierechancen kann also nicht die jeweilige Arbeitsorganisation eröffnen, sondern nur eine andere Organisation.

Bei den neu geschaffenen Juniorprofessuren ergibt sich ein etwas anderes Bild. Zunächst ist es hier möglich, dass interne Kandidaten eine solche Professur übernehmen. Dies kommt auch nicht ganz selten vor. Nach der Studie von Federkeil / Buch (2007) sind immerhin 20\% der befragten Juniorprofessoren an der Hochschule berufen worden, an der sie promoviert wurden, und dies obwohl sie diese Hochschule nach der Promotion nicht bzw. nicht länger als zwei Jahre verlassen hatten. Für die Juniorprofessuren ergibt sich daraus zumindest zum Teil eine interne Karriere in der Universität. Das Problem für diese intern Berufenen ist allerdings, dass ein Tenure-Track-Verfahren ausgeschlossen ist. Dies liegt daran, dass Juniorprofessoren nicht auf eine W2- oder W3-Professur an der gleichen Universität berufen werden können, wenn sie nicht an einer anderen Universität promoviert wurden bzw. nicht mindestens mehrere Jahre

7 Dies folgt daraus, dass es hier keine Unterscheidung in der Benennung gibt. C3 / W2 und C4 / W3 tragen jeweils den Professorentitel und eben nicht den jeweiligen Zusatz.

8 Ein formal striktes Hausberufungsverbot war in der Bundesrepublik nie vorhanden, weil dies auch gegen Artikel 33 Abs. 2 des Grundgesetzes verstoßen würde, der für die Besetzung von öffentlichen Ämtern als Auswahlkriterien lediglich Eignung, Befähigung und fachliche Leistung vorsieht. Unter dem Hausberufungsverbot wird vielmehr formal die bereits in der ersten Fassung des HRG von 1976 vorhandene Formulierung des $\S 45$ Abs. 2 verstanden, nach der nur in begründeten Ausnahmefällen die Berufung eines Mitglieds der eigenen Hochschule möglich war. Mittlerweile ist diese Regelung im HRG nicht mehr vorhanden, sondern die Landeshochschulgesetze regeln diesen Sachverhalt. In allen Landeshochschulgesetzen finden sich entweder die alte Formulierung des HRG oder aber geringfügig abweichende Formulierungen. Substantielle Änderungen sind für Professoren und wissenschaftliche Mitarbeiter nicht vorhanden, sondern nur für Juniorprofessuren (vgl. hierzu Fn. 9+10). Typische Formulierungen finden sich z.B. in den Landeshochschulgesetzen von Bayern ( 18 Abs. 4), Baden-Württemberg (§ 48 Abs. 3) oder Thüringen (§ 78 Abs. 4). 
außerhalb der berufenden Hochschule wissenschaftlich tätig waren. ${ }^{9}$ Für diejenigen Juniorprofessoren, die ihre Promotion an einer anderen Hochschule erworben haben bzw. zumindest mehrere Jahre an einer anderen Hochschule beschäftigt waren, gilt diese Einschränkung hingegen nicht. Hier könnten die Universitäten interne Karrieren für ihre Juniorprofessoren ermöglichen. ${ }^{10}$ Allein die Zahlen zeigen, dass nur wenige Universitäten von der Möglichkeit des Tenure-Track Gebrauch machen. Nur 8\% der in der Studie von Federkeil / Buch (2007) befragten Juniorprofessoren verfügen über ein Tenure-Track im eigentlichen Sinn, also die Möglichkeit, eine Professur ohne öffentliche Ausschreibung zu erhalten. Bei weiteren 12\% wird zumindest eine Bewerbung auf eine öffentlich ausgeschriebene Professur der Universität ermöglicht.

Dass die große Mehrheit der Juniorprofessoren zum einen nicht aus der eigenen Universität rekrutiert wird und zum anderen keine Tenure-Track-Option besitzt, zeigt dann auch die große informell-normative Wirkung des deutschen Hausberufungsverbots. Aber selbst wenn diese Normen sich im Zeitverlauf abschwächen sollten, bleibt ein weiteres Problem: Es gibt insgesamt recht wenig Juniorprofessuren. Nach den letzten Zahlen waren 2008 in den Universitäten lediglich 897 Juniorprofessuren tätig. Hingegen schrumpften die traditionellen Habilitationsstellen der C-Besoldungsstufen (C1-, C2-Assistenten und Dozenten) seit 1998 um fast 10.000 Stellen (Statistisches Bundesamt 2008: 31: 48 f.). Diese Stellen wurden mehrheitlich in wissenschaftliche Mitarbeiterstellen ohne weiteren Zusatz umgewandelt, was bedeutet, dass die Mehrheit der potentiellen W2- und W3-Professoren sich nicht auf einer Juniorprofessorenstelle befindet. Für den Personenkreis der wissenschaftlichen Mitarbeiter gilt aber nach wie vor das in Fn. 8 beschriebene rechtliche Hausberufungsverbot. Hinzu kommt bei diesem Personenkreis - abgeschwächt sicherlich auch für Juniorprofessoren -, dass die informelle Norm, keine Hausberufungen vorzunehmen, weiterhin besteht. Daraus ergibt sich dann ein zwar nicht formal vorgeschriebenes Hausberufungsverbot, aber ein faktisch wirksames.

All dies führt zu der Einschätzung, dass zumindest bisher beim Übergang von der Juniorprofessur bzw. einer Assistenten- oder wissenschaftlichen Mitarbeiterstelle auf eine W2- oder W3-Professur für die Organisationsführung kein relevanter Aufbau von Personalmacht stattgefunden hat. Noch immer ist der absolute Regelfall, dass bei der Erstberufung ein Universitätswechsel vorgenommen werden muss. So spricht auch Musselin (2010: 188) im Hinblick auf das deutsche akademische Karrieresystem zusammenfassend davon, dass es sich hier um ein System handelt, in dem ganz eindeutig ,external labor markets“ dominieren und deutsche Universitäten ,no opportunities of internal career advancement“ zu bieten haben.

Etwas anders stellt sich die Situation bei den weitaus meisten Positionen unterhalb der Professur dar. Hier ist grundsätzlich ein Aufstieg auch innerhalb der Organisation von einem wissenschaftlichen Mitarbeiter der Prädoc- zu einem Mitarbeiter der Postdoc-Phase möglich und auch nicht gerade unüblich. Vielmehr ist hier zu konstatieren, dass es einen internen Arbeitsmarkt gibt, der starke Züge von Patronage aufweist. Dies wird bereits darin deutlich, dass die Professoren ihre wissenschaftlichen Mitarbeiter für die Promotionsphase meist aus dem

9 Diese Regelung wurde bereits bei der Einführung der Juniorprofessur bei der 5. Novelle in $\S 45$ Abs. 2 geregelt. Nach der Nichtigerklärung der Regelungen zur Juniorprofessur durch das Bundesverfassungsgericht 2004 haben die Länder Regelungen in ihren Landeshochschulgesetzen verankert. In den Landeshochschulgesetzen finden sich entweder die ursprüngliche Formulierung des HRG oder aber geringfügige Abweichungen (z.B. in der Exaktheit der zeitlichen Vorgabe der Beschäftigung außerhalb der berufenden Hochschule). Typische Formulierungen finden sich z.B. in den Landeshochschulgesetzen von Sachsen-Anhalt (§36 Abs. 3), Nordrhein-Westfalen (§37 Abs. 2) oder Hamburg (\$14 Abs. 4).

10 Zumindest dann, wenn die Landeshochschulgesetze dies umfassend zulassen. Dies ist nicht immer der Fall, da häufig auch in Bezug auf die Berufung von Juniorprofessoren auf Professorenstellen die Formulierung eines „Ausnahmefalles“ vorhanden ist. 
Pool der ihnen bekannten Studenten rekrutieren. Die Rekrutierung der „Assistenten“ ist ebenfalls durch persönliche Bekanntheit geprägt, wobei häufig Mitarbeiter des eigenen Lehrstuhls zum Zuge kommen (Enders 2008). Während also für die Professoren von einem externen Arbeitsmarkt auszugehen ist, findet sich unterhalb dieser Position im deutschen Hochschulsystem eher ein interner Markt.

Festzuhalten ist, dass auf Lehrstuhlebene durchaus Personalmacht existiert. Da diese aber von den einzelnen Professoren ausgeübt wird, ist sie kein Indikator für die in Teil 2 beschriebene Akteurswerdung von Universitäten. Im Gegenteil: Der Professor entscheidet darüber, ob und unter welchen Bedingungen er einen wissenschaftlichen Mitarbeiter weiterbeschäftigt, während weder andere Professoren noch der Institutsleiter oder der Dekan oder gar die Hochschulleitung hier einen direkten Einfluss haben. All dies ist Ausdruck des traditionellen Lehrstuhlprinzips an deutschen Universitäten (Kreckel 2008 a: 50), wobei sich am System der Rekrutierung seit der Ordinarienuniversität kaum etwas verändert hat.

Die Macht der Professoren gegenüber den Lehrstuhlmitarbeitern ist sogar größer als die Macht eines Abteilungsleiters in Wirtschaftsorganisationen und Verwaltungen. Dies liegt daran, dass die Abhängigkeit der Mitarbeiter sich nicht nur auf die Frage der Weiterbeschäftigung bezieht, sondern auch auf die Weiterqualifikation und somit dem Verbleib im wissenschaftlichen System. Sowohl für die Promotion als auch für die Habilitation ist die Unterstützung des Professors unabdingbar. Die in Deutschland traditionell wenig strukturierte Promotions- und Habilitationsphase mit dem immer noch vorherrschenden „Meister-Schüler-Modell“ hat dann nicht nur Auswirkungen auf das Alter, die Art der Betreuung usw., sondern auch auf die Machtverhältnisse innerhalb der Universität. Durch den Aufbau von Graduiertenkollegs wird zwar versucht, die Doktorandenausbildung stärker auf Instituts- oder Fakultätsebene zu verlagern, noch immer promoviert aber der weitaus größte Teil an einem Lehrstuhl (Bosbach 2009). Für die wissenschaftlichen Mitarbeiter an Lehrstühlen ergibt sich dann auch eine Bindung an den jeweiligen Professor und nicht an die Organisation. Um es klar zu sagen: Der Professor hat etwas zu bieten, nicht die Universität.

Insgesamt ist zu konstatieren, dass die Universitätsführung an deutschen Universitäten kaum einen Einfluss auf den operativen Kern über die Personalmacht ausüben kann. Sie kann weder Karrieren verhindern, noch kann sie welche bieten.

In fast allen anderen nationalen Universitätssystemen ist dies anders. ${ }^{11}$ Entweder es gibt geordnete interne Karrieren innerhalb einer Universität oder aber es wird zumindest auf ein Hausberufungsverbot verzichtet bzw. eine solche Regelung wird nicht wie in Deutschland auch stark informell-normativ abgestützt. Das betrifft dann nicht nur die Professoren, sondern auch die weitaus meisten anderen Beschäftigten im wissenschaftlichen Bereich der Universitäten. Dies liegt auch daran, dass viele europäische Hochschulsysteme mittlerweile das traditionelle Lehrstuhlprinzip zugunsten eines Fakultätsprinzips ausgetauscht haben (Enders 2000 b), bei dem die Fakultät über die Karriere der wissenschaftlichen Mitglieder entscheidet und nicht der jeweilige Lehrstuhlinhaber (zu beiden Prinzipien Neave / Rhoades 1987: 214ff.).

In Bezug auf geordnete Karrieren ist z.B. an die gestuften Aufstiege vom Assistant zum Associate und schließlich zum Full Professor des amerikanischen Systems zu denken (Musselin 2010: 183ff.). Auch in Großbritannien gibt es traditionell einen internen Aufstieg vom Lecturer zum Reader und schließlich zum Professor. Auch Schweden hat 1999 interne Karrieren geschaffen, wobei die Universitäten sogar verpflichtet wurden, ihrem Personal Entwicklungsmöglichkeiten zu bieten. Dies strukturiert und verbessert die Aufstiegsaussichten

11 Vgl. hierzu nur die umfangreichen Monographien von Kreckel (2008 a) und Musselin (2010), in denen das deutsche akademische Karrieresystem mit anderen europäischen Systemen und dem der USA verglichen wird. 
nicht nur, sondern die Wissenschaftler werden ,more dependent on the staffing and promotion policy of their own institution and their own department" (Askling 2000: 219).

Die Wirkung eines fehlenden formal und informell wirksamen Hausberufungsverbots kann beispielhaft an Frankreich und Italien gezeigt werden. Für Frankreich beschreibt Musselin zwar, dass interne Rekrutierungen als „not legitimate“ betrachtet werden, aber 55\% der französischen Professoren zuvor an der gleichen Universität eine unbefristete Assistenzprofessur innehatten (Musselin 2005: 140). Capano berichtet für Italien, dass trotz eines offiziellen Wettbewerbssystems zwischen 1998 und 2005 95\% der Full-Professoren und 75\% der Associate-Professoren von der gleichen Universität eingestellt wurden, für die sie auch vorher gearbeitet hatten (Capano 2008).

Interne Karrieren zur Professur sind daraus folgend in einer Reihe von anderen nationalen Universitätssystemen formal oder informell implementiert, sie werden auf jeden Fall nicht wie in Deutschland durch das Zusammengreifen von rechtlichen und informellen Normen verhindert. Ist eine solche interne Karriere möglich, werden diejenigen Wissenschaftler, die eine solche anstreben, aber auch einer organisationalen Logik unterworfen. Dies folgt daraus, dass sich in aller Regel eine Beteiligung organisationaler Stellen (Hochschulleitung, Dekane) bei der Entscheidung über die Karriere innerhalb der Organisation finden lässt (vgl. die Länderberichte in Enders 2000 a sowie Musselin 2010 mit Bezug auf Frankreich und die USA). Die Anpassungsbereitschaft an die Regeln und Normen der Organisation dürfte in diesen Systemen dann auch deutlich höher sein, weil die Organisation Karrieren bieten bzw. verwehren kann.

Die wirksame Verhinderung von Karrieren innerhalb der Universitäten in Deutschland ist auch im Vergleich zu anderen Professionsorganisationen ein Alleinstellungsmerkmal. Zwar weist die soziologische Professionstheorie darauf hin, dass Professionelle im Gegensatz zu anderen Berufsgruppen eher Karrieren zwischen Organisationen verfolgen (Scott 1968: 212), dies heißt aber keineswegs, dass Professionsorganisationen nicht versuchen, diese Logik zumindest teilweise zu durchbrechen. So sind interne Karrieren von Ärzten in Krankenhäusern durchaus üblich. Gleiches gilt für Großkanzleien mit ihren ausgeprägten internen Karrieremustern (Heinz et al. 2005: 140ff.). Der vollkommene Verzicht auf dieses organisationale Machtinstrument im deutschen Universitätssystem ist demnach weder im internationalen Vergleich der Universitätssysteme noch im Vergleich zu anderen Professions-, Wirtschafts- und Verwaltungsorganisationen typisch.

\section{Organisationsmacht}

In den meisten Organisationen existiert neben der Personalmacht noch eine weitere fundamentale Machtquelle, die auf der Ausschlussmöglichkeit der Mitglieder beruht: die Organisationsmacht. Organisationsmacht ist nach Luhmann (1975: 104ff.) dadurch gekennzeichnet, dass Organisationen die Möglichkeit haben, Mitglieder auszuschließen, wenn diese gegen die Mindestanforderungen der Organisation verstoßen. Mindestanforderungen sind dabei die Anerkennung der formalen Organisationsregeln oder das Unterlassen von Handlungen, die offensichtlich den Organisationszielen zuwiderlaufen. Organisationsmacht wird in der Regel von Vorgesetzten ausgeübt. Hierbei ist zu beachten, dass die tatsächliche Anwendung dieser Macht sowohl für das Mitglied als auch für den Vorgesetzten mit erheblichen Transaktionskosten verbunden ist und deshalb für beide eine Vermeidungsalternative darstellt. Die Wirkung der Organisationsmacht liegt deshalb weniger in der tatsächlichen Anwendung, sondern im latenten Mitlaufen der Drohung der Anwendungsmöglichkeit im Hintergrund. Immer dann, wenn eine solche Drohung nicht glaubhaft ist oder gemacht werden kann, kommt es demzufolge zu einer Schwächung dieser Machtquelle. 
Im Hinblick auf die Professoren an den deutschen Universitäten sollte deutlich sein, dass ein Rückgriff auf die Organisationsmacht von Seiten der Hochschulleitung kaum möglich ist, weil es keine Verfahren gibt ,how a professor could be fired even if he or she is lazy, incompetent or no longer needed“" (Dilger 2007: 109).

Dies liegt zunächst daran, dass die weitaus meisten Professoren Beamte auf Lebenszeit sind. Darüber hinaus ist immer auch die in Art. 5 Abs. 3 Grundgesetz verfassungsrechtlich garantierte Freiheit von Forschung und Lehre zu beachten. Diese Freiheit ist in der deutschen Tradition eng verknüpft mit der Unabhängigkeit der Professoren (Baker / Lenhardt 2008), was ebenfalls die Stellung der Professoren gegenüber der Organisation absichert. Diese institutionellen Absicherungen erschweren letztendlich die Entlassung eines Professors erheblich, was dazu führt, dass eine glaubhafte Androhung des Organisationsausschlusses nicht möglich ist. Die Organisationsführung ist dann darauf angewiesen, riskante Gerichtsverfahren zur Durchsetzung der Organisationsmacht zu führen, was angesichts des hohen Zeit- und Personalaufwandes sowie des ungewissen Ausgangs nur extrem selten vorkommt.

Auch die neueren Universitätsreformen haben kaum etwas an der traditionellen Schwäche dieser Machtquelle verändert. Nach wie vor ist der auf Lebenszeit ernannte Professor das Regelmodell in allen Landeshochschulgesetzen. Einzig die Regelungen in einigen Landeshochschulgesetzen, die erste Professur zu befristen bzw. nur auf Probe zu gewähren, stören das traditionelle Bild. In den Ländern ${ }^{12}$ mit Befristungsregel kann deshalb gegenüber einem begrenzten Personenkreis zumindest zu einem vorher festgelegten Zeitpunkt Organisationsmacht ausgeübt werden. Hier ist demnach eine vorsichtige Aktivierung der Machtquelle zu konstatieren. Ob sie wirklich flächendeckend genutzt wird, bleibt allerdings abzuwarten. Dies auch deshalb, weil sowohl die Anstellung eines Professors im Angestelltenverhältnis wie auch die Professur auf Zeit bzw. auf Probe grundsätzlich auch schon durch die Regelungen des HRG von 1976 abgedeckt gewesen wären (Detmer 2004: 50). Die vorhandenen Regelungen wurden aber nicht genutzt, was zumindest auch für die neuen Regelungen nicht auszuschließen ist.

Insgesamt ergibt sich für alle Bundesländer, dass die Organisationsmacht als Sanktionsmöglichkeit der Hochschulleitung gegenüber der großen Mehrheit der Professoren nach wie vor kaum eine Rolle spielt. Insbesondere ist sie nicht so ausgestaltet, dass sie im Hintergrund als latente Drohung mitlaufen könnte.

Die Organisationsebene hat aber nicht nur gegenüber den Professoren keine Organisationsmacht, sondern auch gegenüber dem größten Teil des weiteren wissenschaftlichen Personals. Wie schon bei der Personalmacht ist hier vielmehr festzustellen, dass Organisationsmacht in Bezug auf die Lehrstuhlmitarbeiter bei den Professoren konzentriert ist. Professoren, nicht Dekane oder Hochschulleitungen, ,hire and potentially fire“(Dilger 2007: 103) wissenschaftliche Mitarbeiter und entscheiden somit auch über Aufnahme und Ausschluss von Organisationsmitgliedern. ${ }^{13}$ Dass Professoren und nicht Institute oder Fakultäten „Gate-Keeper" der wissenschaftlichen Karriere bis zur Professur sind, folgt gleichfalls aus dem traditionellen deutschen Lehrstuhlprinzip mit seiner Betonung der Unabhängigkeit des Professors.

12 Eine Sollvorschrift findet sich in den Bundesländern Brandenburg, Hessen, Schleswig-Holstein und Thüringen. Eine Befristung kann in Baden-Württemberg, Hamburg, Niedersachsen, Sachsen und dem Saarland erfolgen. In Bremen ist eine Befristung möglich, wenn die Hochschule und der Senator für Wissenschaft dies im Einvernehmen festlegen. In Bayern muss hingegen eine Befristung bei der Erstberufung dann erfolgen, wenn der Kandidat bisher keine drei Jahre an einer Hochschule hauptberuflich tätig war.

13 Formal begrenzt wird die Organisationsmacht hier allerdings durch die Befristungsregeln des Wissenschaftszeitgesetzes. 
Der Mangel an Organisationsmacht in deutschen Universitäten ist dabei im internationalen Vergleich keinesfalls ein stabiles Muster. Zunächst ist der Beamtenstatus - und damit die generelle Unkündbarkeit der Professoren - in vielen anderen Ländern nicht vorhanden oder wurde, wie z.B. in Schweden (Burkhardt 2008), abgeschafft. Die Professoren haben zwar dann nicht befristete Verträge, bleiben aber kündbar. Auch in den Niederlanden und Großbritannien ist der Ausschluss von Professoren möglich. Es zeigt sich gerade in diesen beiden Ländern, in denen die Umsetzung des NPM-Modells besonders weit fortgeschritten ist, dass im Reformverlauf die Möglichkeit geschaffen und auch genutzt wurde, Professoren zu entlassen (de Weert 2000; Fulton 2000). Unabhängig davon, wie begründet diese Entlassungen waren, verstärken sie im Reformprozess aber das Steuerungspotential durch Organisationsmacht, weil sie eine glaubhafte Androhung der Nutzung ermöglichen bzw. erleichtern.

Das Vorherrschen von Tenure-Positionen für Professoren an amerikanischen Hochschulen, die ihnen eine für das amerikanische Beschäftigungssystem untypisch hohe berufliche Absicherung gewährleisten, ist in den letzten Jahren erheblich kritisiert worden (Musselin 2010: $27 \mathrm{f}$.). Auch hier ist zu konstatieren, dass es zu einer Verstärkung der Organisationsmacht gekommen ist. So zeigen die Zahlen, dass insgesamt das Tenure-Track-System zurückgefahren wurde und stattdessen an den Universitäten vermehrt Wissenschaftler befristet eingestellt wurden, die auch leichter wieder ausgeschlossen werden können. ${ }^{14}$ Während demnach in den 1970er Jahren in den Universitäten in den USA noch sehr viel stärker auf Personalmacht und entsprechende Karriereanreize gesetzt wurde, wird nun der Einsatz von Organisationsmacht erleichtert. Ähnliche Tendenzen sind auch für die britischen Universitäten vorhanden (Kreckel 2008 b) und hier in Zusammenhang mit der finanziellen Unsicherheit aufgrund der seit den 1980er Jahren eingeführten leistungsabhängigen Mittelvergabe entstanden. Die Universitäten reagieren auf diese Unsicherheit damit, dass sie den Anteil an festangestellten Mitarbeitern deutlich verringern und deshalb auch keine Tenure-Track-Optionen gewähren (Fulton 2000).

Der Verzicht auf Organisationsmacht ist aus diesen Betrachtungen heraus keineswegs ein typisches Muster in anderen Universitätssystemen. In Deutschland wird demgegenüber auf eine Machtquelle der Organisationsführung verzichtet, die in anderen nationalen Systemen zur Verfügung steht. Abschließend wollen wir uns der Frage zuwenden, welche Perspektiven sich hieraus ergeben und ob es möglicherweise Alternativen zur Verfügbarkeit über Organisationsund Personalmacht gibt.

\section{Abschließende Überlegungen}

Wie wir versucht haben zu zeigen, gibt es zwar einen generellen Trend in Richtung auf so genannte „,complete organizations“, jedoch ist die Umsetzung in Bezug auf die Stärkung der internen Hierarchie in Organisationen auf spezifische Machtpotentiale angewiesen, die wir für den Fall deutscher Universitäten nicht aufspüren konnten. Dies ermöglicht nicht nur die in Teil 3 aufgezeigten internationalen Vergleichsperspektiven, sondern auch die zwischen Organisationstypen. Die beiden für die Einflussnahme auf das Verhalten der Mitglieder typischen und dominierenden Machtquellen in Organisationen - die mit Karrierechancen lockende Personalmacht und die mit dem Ausschluss aus der Organisation drohende Organisationsmacht

14 Der Anteil der Wissenschaftler mit Tenure ist solchermaßen in den USA von 65\% im Jahr 1980 / 81 auf 49\% im Jahr 2007 / 2008 gesunken (vgl. National Center for Education Statistics (NCES). Chait (2002: 19) zufolge ist es insbesondere zu einem dramatischen Anstieg von Teilzeitprofessuren in den USA gekommen, deren Anteil an den Professuren insgesamt sich zwischen 1970 und 1995 von 22\% auf $41 \%$ nahezu verdoppelt hat. Diese Tendenz zeigt sich der Analyse von Donoghue (2008) zufolge besonders dramatisch im Hinblick auf Fächer, die wie die Geisteswissenschaften keinen unmittelbaren ökonomischen Nutzen erwarten lassen, sowie im Hinblick auf Hochschulen, die gewinnorientiert und / oder rein ausbildungsorientiert sind. 
- sind in deutschen Universitäten in Bezug auf den operativen Kern und die in ihm tätigen Wissenschaftler erheblich eingeschränkt. Beide Machtquellen sind insbesondere nicht so ausgestaltet, dass ihre Nutzungsmöglichkeit von den Mitgliedern quasi automatisch antizipiert wird und hierdurch ihr Verhalten beeinflusst. Dies ist in anderen institutionellen Settings deutlich anders. Während in Wirtschaftsorganisationen zumeist beide Machtquellen zur Verfügung stehen, besteht in deutschen Verwaltungen sowie in anderen nationalen Universitätssystemen vor allem die Möglichkeit, auf Personalmacht zu setzen; auch Organisationsmacht steht als Machtquelle in anderen Universitätssystemen zunehmend zur Verfügung. Aufgrund des Fehlens dieser Machtquellen können sich deutsche Universitäten folglich nur begrenzt in Richtung der in Teil 2 dargestellten „complete organization“ im Sinne von Brunsson und Sahlin-Andersson (2000) entwickeln. Wird berücksichtigt, dass der Koordinationsmechanismus der Hierarchie ohne Sanktionspotentiale nicht angewendet werden kann, so stellt sich die Frage, ob hier nicht eine bisher kaum beachtete und spezifisch deutsche Barriere für die Einführung des NPM-Modells vorliegt.

Diskutiert werden muss aber auch, ob die deutschen Universitäten womöglich funktionale Äquivalente zu den typischen Machtquellen besitzen. Insbesondere die neueren Veränderungen in Bezug auf die Leistungsbesoldung und die befristete Mittelzusage könnten hier eine Rolle spielen. Diese Frage wollen wir abschließend kurz behandeln.

Ganz grundsätzlich ist darauf hinzuweisen, dass beide Reformmaßnahmen das Macht- und Sanktionspotential der Organisationsführung stärken. Unserer bisherigen Argumentation folgend besteht die Neuartigkeit aus organisationssoziologischer Sicht vor allem darin, dass die Gewährung oder der Entzug von Ausstattung und Leistungsbesoldung insgesamt an die Universität als Organisation delegiert wurde, also nicht mehr wie im traditionellen System Gegenstand von Verhandlungen zwischen Professor und Ministerium sind. Wir sehen hierin einen wichtigen Indikator für den Übergang der losen gekoppelten Universitätsorganisation - „ohne Zentrum und ohne Spitze“, um hier die Luhmannsche Charakterisierung der modernen, funktional differenzierten Gesellschaft zur Beschreibung der traditionellen Universitätsorganisation aufzugreifen - zu einer „complete organization“, die über eigenständige, handlungs- und entscheidungsfähige sowie mit Machtquellen ausgestattete Leitungsebenen verfügt.

Allerdings sind bei der Leistungsbesoldung und der befristeten Mittelzusage auch Probleme erkennbar. Erstens ist darauf hinzuweisen, dass beide organisationalen Machtpotentiale nur bestimmte Mitgliedergruppen umfassen. Für die neue Dynamik der Gehalts- und Ausstattungsstrukturen gilt zunächst, dass sie sich nur auf Professoren beziehen, die anderen wissenschaftlichen Beschäftigten hingegen nicht erfassen. Ihre Reichweite im Hinblick auf das hier diskutierte Verhältnis von Hochschulorganisationen und ihren wissenschaftlichen Mitarbeitern bleibt demnach begrenzt. Zweitens unterliegt die Nutzung von variablen Gehalts- und Ausstattungsstrukturen spezifischen Wirkungsgrenzen, die sich vor allem auf fachlich-disziplinäre Unterschiede beziehen. Im Hinblick auf die Leistungsbesoldung dürften Professoren, die außerhalb der Universitäten relevante Einkommensquellen haben, von diesem Anreizmechanismus nicht oder doch kaum betroffen sein. Hier ist insbesondere an die Medizin, die Rechtswissenschaft oder die Ingenieurwissenschaften zu denken. Ebensolche Unterschiede gelten auch hinsichtlich der befristeten Mittelzusage für Ausstattungsfragen. Klassische geisteswissenschaftliche Fächer, in denen die Forschung weitgehend Individualforschung ist, sind hiervon unabhängiger als stärker vernetzte Wissenschaften, die, wie weite Teile der Naturwissenschaften, auf eine erhebliche personelle und materielle Ausstattung angewiesen sind, um überhaupt Forschung durchführen zu können (Jansen et al. 2007). Vertreter derartiger Fächer oder Forschungsrichtungen sind jedoch besonders aktiv in der Einwerbung von Drittmitteln, was sie wiederum unabhängiger von Ausstattungsgewinnen durch die Organisation macht. Dies auch deshalb, weil die Ausstattungsgewinne durch Einwerbung von Drittmitteln 
deutlich höher liegen dürften als Ausstattungsgewinne über die jeweilige Universität. Hinzu kommt, dass zwar Drittmittel auch als Indikator für die befristete Mittelzusage genutzt werden, letztlich aber andere Organisationen über deren Bewilligung entscheiden, was dazu führt, dass andere Organisationen einen erheblichen Einfluss auf die Anwendung der Sanktionsmöglichkeiten der jeweiligen Universitätsorganisation haben. Drittens unterliegt die befristete Mittelzusage rechtlichen Grenzen, da es ,nach wie vor unstrittig“" (Seidler 2004: 503) ist, dass Professoren, aber auch wissenschaftliche Einrichtungen, aufgrund der Wissenschaftsfreiheit ein Recht auf eine Grund- und Mindestausstattung haben. Die Höhe dieser Ausstattung ist zwar abstrakt nicht zu beziffern, bildet aber eine verfassungsrechtliche Grenze des organisationalen Machtpotentials durch Mittelverteilung.

Hinzu kommt, dass die Reformen bisher einseitig auf eine Stärkung der Organisationsebene über die Verteilung von Finanzen ausgerichtet sind und ökonomisches Kapital zum dominanten Steuerungsmedium wird. Dagegen kann zunächst eingewendet werden, dass im Wissenschaftssystem Geld das falsche Steuerungsmedium ist, weil es nicht fähig ist, auf das Verhalten der Wissenschaftler bzw. die Selbstorganisation der Wissenschaft einzuwirken (Minssen / Wilkesmann 2003; Luhmann 1990). Sollte diese Einwirkung aber dennoch gelingen, dann entsteht das Problem, dass nicht absehbar ist, welche möglicherweise problematischen Effekte hieraus resultieren. So wird häufig vermutet, dass Professoren auf die neuen extrinsischen Motivationsanreize mit einem langfristig für die Wissenschaft schädlichen Verlust ihrer intrinsischen Motivationsquellen reagieren (z.B. Zauner 2005: 190).

Zusammengefasst lässt sich also sagen, dass die neu geschaffenen Anreizmechanismen nicht den gesamten wissenschaftlichen Kern der Universität umfassen, nicht alle Professoren in gleicher Weise treffen, rechtlichen Grenzen unterliegen und einseitig auf Geld als Steuerungsmedium setzen. Im Gegensatz zur Organisations- und Personalmacht können sie deshalb nicht als basale und umfassende Machtpotentiale der Organisation angesehen werden, da die große Mehrheit der Mitglieder gerade nicht quasi automatisch und relativ unabhängig von anderen Faktoren erfasst wird.

Dies wird auch durch einen Vergleich mit anderen Hochschulsystemen, Wirtschaftsorganisationen und Organisationen der öffentlichen Verwaltung deutlich. Hier wird erkennbar, dass die Höhe des Gehaltes und die Ausstattung die Organisations- und Personalmacht eher flankieren, aber nicht fähig sind, diese im Sinne funktionaler Äquivalente zu ersetzen. Relativ zu anderen Universitätssystemen, Verwaltungs- und Wirtschaftsorganisationen ist deshalb zu konstatieren, dass die Organisationsebene der deutschen Universitäten in Bezug auf Karrieren und Beschäftigungsbedingungen auch nach den bisherigen Reformen vergleichsweise schwach bleibt. Im Gegensatz zu Diskussionen um das New Public Management und der in diesem Rahmen angenommenen Konstruktion von ,complete organizations“, wie sie in Teil 2 dargestellt wurden, erscheint die deutsche Universität im Hinblick auf die wissenschaftliche Karriere und die Beschäftigungsbedingungen deshalb immer noch als Organisation mit sehr spezifischen Eigenarten. Dies wirft dann aber die Frage auf, ob eine Übertragung von Steuerungsinstrumenten aus der Wirtschaft unter diesen Bedingungen überhaupt möglich ist. Insbesondere im Hinblick auf eine hierarchische, auf Personal- und Organisationsmacht basierende Steuerung innerhalb der Universitäten sind erhebliche Einschränkungen sichtbar geworden. Die Ausführungen zeigen deshalb auch, dass im Hinblick auf die in der allgemeinen Governanceliteratur diskutierten Governancemechanismen, wie hier am Beispiel der Hierarchie gezeigt, die institutionellen Voraussetzungen jeweils zu prüfen sind und nicht als gegeben vorausgesetzt werden können.

Hinzuweisen ist noch darauf, dass die von uns eingenommene Perspektive strikt organisationssoziologisch war und eine normative Einschätzung der Wünschbarkeit bzw. NichtWünschbarkeit des Einsatzes von Machtpotenzialen nicht im Vordergrund stand. Vielmehr 
ergibt sich hier eine offene Frage, die ebenso über unseren Beitrag hinausweist, wie die nicht nur auf Organisations- und Personalmacht zu beschränkende Diskussion des Themas „Macht in Hochschulorganisationen“".

\section{Literaturver}

Altbach, Philip G. (1991): University Reform, in: Philip G. Altbach (Hrsg.), International Higher Education. An Encyclopedia, New York - London, S. 261-274.

Askling, Berit (2000): Academic Staff in Sweden, in: Jürgen Enders (Hrsg.), Employment and Working Conditions of Academic Staff in Europe, Frankfurt / Main, S. 210-223.

Baker, David P. / Gero Lenhardt (2008): The Institutional Crisis of the German Research University, in: Higher Education Policy 21, S. 49-64.

Blümel, Albrecht / Katharina Kloke / Georg Krücken (2010): Professionalisierungsprozesse im Hochschulmanagement in Deutschland, in: Andreas Langer / Andreas Schroer (Hrsg.), Professionalisierung im Nonprofit Management, Wiesbaden, S. 105-127.

Bogumil, Jörg / Stephan Grohs / Sabine Kuhlmann / Anna K. Ohm (2007): Zehn Jahre Neues Steuerungsmodell, Berlin.

Bosbach, Eva (2009): Von Bologna nach Boston. Perspektiven und Reformansätze in der Doktorandenausbildung anhand eines Vergleichs zwischen Deutschland und den USA, Leipzig.

Boyer, Ernest / Philip G. Altbach / Mary J. Whitelaw (1994): The Academic Profession. An International Perspective. A Special Report, Princeton.

Braun, Dietmar / François-Xavier Merrien (1999 a): Governance of Universities and Modernisation of the State: Analytical Aspects, in: Dietmar Braun / François-Xavier Merrien (Hrsg.), Towards a New Model of Governance for Universities? A Comparative View, London, S. 9-33.

Braun, Dietmar / François-Xavier Merrien (Hrsg.) (1999 b): Towards a New Model of Governance for Universities? A Comparative View, London.

Brunsson, Nils / Kerstin Sahlin-Andersson (2000): Constructing Organizations: The Example of Public Sector Reform, in: Organization Studies 24, S. 721-746.

Burkhardt, Anke (2008): Schweden, in: Reinhard Kreckel (Hrsg.), Zwischen Promotion und Professur, Leipzig, S. 203-238.

Capano, Giliberto (2008): Looking for Serendipity: The Problematical Reform of Government within Italy's Universities, in: Higher Education 55, S. 481-504.

Chait, Richard P. (Hrsg.) (2002): The Question of Tenure, Cambridge / Mass.

Clark, Burton (1983): The Higher Education System. Academic Organization in Cross-national Perspective, Berkeley.

Clegg, Stewart / David Courpasson / Nelson Phillips (2009): Power and Organizations, Los Angeles.

Cohen, Michael D. / James G. March / Johan P. Olsen (1972): A Garbage Can Model of Organizational Choice, in: Administrative Science Quarterly 17, S. 1-25.

de Boer, Harry / Jürgen Enders / Uwe Schimank (2007 a): On the Way towards New Public Management? The Governance of University Systems in England, the Netherlands, Austria, and Germany, in: Dorothea Jansen (Hrsg.), New Forms of Governance in Research Organizations. Disciplinary Approaches, Interfaces and Integration, Dordrecht, S. 137-152.

de Boer, Harry / Jürgen Enders / Liudvika Leisyte (2007 b): Public Sector Reform in Dutch Higher Education: The Organizational Transformation of the University, in: Public Administration 85, S. 27-46.

de Boer, Harry / Liudvika Leisyte / Jürgen Enders (2006): The Netherlands - 'Steering from a Distance', in: Barbara M. Kehm / Ute Lanzendorf (Hrsg.), Reforming University Governance. Changing Conditions for Research in Four European Countries, Bonn, S. 59-96. 
Detmer, Hubert (2004): Das Recht der Universitätsprofessoren, in: Michael Hartmer / Hubert Detmer (Hrsg.), Hochschulrecht. Ein Handbuch für die Praxis, Heidelberg, S. 46-123.

de Weert, Egbert (2000): Academic Staff in the Netherlands, in: Jürgen Enders (Hrsg.), Employment and Working Conditions of Academic Staff in Europe, Frankfurt / Main, S. 168-180.

Dilger, Alexander (2007): German Universities as State-sponsored Co-operatives, in: management revue 18, S. 102-116.

DiMaggio, Paul J. / Walter W. Powell (1983): The Iron Cage Revisted: Institutional Isomorphism and the Collective Rationality in Organizational Fields, in: American Sociological Review 48, S. 147-160.

Donoghue, Frank (2008): The Last Professor. The Corporate University and the Fate of the Humanities, New York.

Enders, Jürgen (1996): Die wissenschaftlichen Mitarbeiter. Ausbildung, Beschäftigung und Karriere der Nachwuchswissenschaftler und Mittelbauangehörigen an den Universitäten, Frankfurt / Main.

Enders, Jürgen (Hrsg.) (2000 a): Employment and Working Conditions of Academic Staff in Europe, Frankfurt / Main.

Enders, Jürgen (2000 b): Academic Staff in the European Union, in: Jürgen Enders (Hrsg.). Employment and Working Conditions of Academic Staff in Europe. Frankfurt / Main, S. 29-53.

Enders, Jürgen (2008): Professor werden ist sehr schwer, Professor sein dann gar nicht mehr? Ein Betrag zur Personalstrukturreform an den Hochschulen, in: Hildegard Matthies / Dagmar Simon (Hrsg.), Wissenschaft unter Beobachtung. Effekte und Defekte von Evaluationen, Wiesbaden, S. 83-99.

Eulenburg, Franz (1908): Der „Akademische Nachwuchs“. Eine Untersuchung über die Lage und die Aufgaben der Extraordinarien und Privatdozenten, Leipzig - Berlin.

European Review (Hrsg.) (2010): Diversification of Higher Education and the Academic Profession Papers from the Hercules Symposium, in: European Review 18, Supplement S1.

Federkeil, Gero / Florian Buch (2007): Fünf Jahre Juniorprofessur - Zweite CHE-Befragung zum Stand der Einführung, Arbeitspapier Nr. 90, Gütersloh.

Ferlie, Ewan / Christine Musselin / Gianluca Andresani (2008): The Steering of Higher Education Systems: A Public Management Perspective, in: Higher Education 56, S. 325-348.

Fulton, Oliver (2000): Academic Staff in the United Kingdom, in: Jürgen Enders (Hrsg.), Employment and Working Conditions of Academic Staff in Europe, Frankfurt / Main, S. 224-234.

Gornitzka, Ase / Kyvik Svein / Ingvild M. Larsen (1998): The Bureaucratisation of Universities, in: Minerva 36, S. 21-47.

Gross, Christiane / Monika Jungbauer-Gans (2007): Erfolg durch Leistung? Ein Forschungsüberblick zum Thema Wissenschaftskarrieren, in: Soziale Welt 58, S. 453-471.

Hall, Peter A. (1993): Policy Paradigms, Social Learning, and the State: The Case of Economic Policymaking in Britain, in: Comparative Politics 25, S. 275-296.

Hartwig, Lydia (2006): Neue Finanzierungs- und Steuerungsstrukturen und ihre Auswirkungen auf die Universitäten. Zur Situation in vier Bundesländern, in: Beiträge zur Hochschulforschung 28, S. 6-25.

Heinz, John P. / Robert L. Nelsen / Rebecca L. Sandefur / Edward O. Laumann (2005): Urban Lawyers. The New Social Structure of the Bar, Chicago.

Hüther, Otto (2009): Hochschulräte als Steuerungsakteure, in: Beiträge zur Hochschulforschung 31, S. 50-73.

Hüther, Otto (2010): Von der Kollegialität zur Hierarchie? Eine Analyse des New Managerialism in den Landeshochschulgesetzen, Wiesbaden.

Jaeger, Michael (2006): Leistungsorientierte Budgetierung: Analyse der Umsetzung in ausgewählten Universitäten und Fakultäten / Fachbereichen. HIS Kurzinformation, Hannover. 
Jansen, Dorothea / Andreas Wald / Karola Franke / Ulrich Schmoch / Torben Schubert (2007): Drittmittel als Performanzindikator der wissenschaftlichen Forschung. Zum Einfluss von Rahmenbedingungen auf Forschungsleistungen, in: Kölner Zeitschrift für Soziologie und Sozialpsychologie 59, S. 125-149.

Joas, Hans / Michael Bochow (1987): Wissenschaft und Karriere. Der berufliche Verbleib des akademischen Mittelbaus, Frankfurt / Main.

Kehm, Barbara M. / Ute Lanzendorf (2006 a): Germany - 16 Länder Approaches to Reform, in: Barbara M. Kehm / Ute Lanzendorf (Hrsg.), Reforming University Governance. Changing Conditions for Research in Four European Countries, Bonn, S. 135-185.

Kehm, Barbara M. / Ute Lanzendorf (Hrsg.) (2006 b): Reforming University Governance. Changing Conditions for Research in Four European Countries, Bonn.

Klecha, Stephan / Wolfgang Krumbein (Hrsg.) (2008): Die Beschäftigungssituation von wissenschaftlichem Nachwuchs, Wiesbaden.

Krais, Beate (2000): Wissenschaftskultur und Geschlechterordnung. Über die verborgenen Mechanismen männlicher Dominanz in der akademischen Welt, Frankfurt / Main.

Kosmützky, Anna (2010): Von der organisierten Institution zur institutionalisierten Organisation? Eine Untersuchung der (Hochschul-)Leitbilder von Universitäten. Unveröffentlichte Dissertation, Universität Bielefeld, Fakultät für Soziologie.

Kreckel, Reinhard (2008 a): Zwischen Promotion und Professur. Das wissenschaftliche Personal in Deutschland im Vergleich mit Frankreich, Großbritannien, USA, Schweden, den Niederlanden, Österreich und der Schweiz, Leipzig.

Kreckel, Reinhard (2008b): Großbritannien, in: Ders. (Hrsg.), Zwischen Promotion und Professur, Leipzig, S. 124-165.

Krücken, Georg / Albrecht Blümel / Katharina Kloke (2010): Hochschulmanagement - auf dem Weg zu einer neuen Profession?, in: WSI-Mitteilungen 5, S. 234-241.

Krücken, Georg / Anna Kosmützky / Mark Torka (Hrsg.) (2007): Towards a Multiversity? Universities between Global Trends and National Traditions, Bielefeld.

Krücken, Georg / Frank Meier (2006): Turning the University into an Organizational Actor, in: Gili S. Drori / John W. Meyer / Hokyu Hwang (Hrsg.), Globalization and Organization. World Society and Organizational Change, Oxford, S. 241-257.

Lange, Stefan (2009): Hochschulräte, in: Stefan Hornbostel / Andreas Knie / Dagmar Simon (Hrsg.), Handbuch Wissenschaftspolitik, Wiesbaden, S. 347-360.

Lange, Stefan / Uwe Schimank (2007): Zwischen Konvergenz und Pfadabhängigkeit: New Public Management in den Hochschulsystemen fünf ausgewählter OECD-Länder, in: Katharina Holzinger/Helge Jörgen / Christoph Knill (Hrsg.), Transfer, Diffusion und Konvergenz von Politiken, Wiesbaden, S. 522-548.

Leisyte, Liudvika / Harry de Boer / Jürgen Enders (2006): England - Prototype of the 'Evaluative State', in: Barbara M. Kehm / Ute Lanzendorf (Hrsg.), Reforming University Governance. Changing Conditions for Research in Four European Countries, Bonn, S. 21-56.

Luhmann, Niklas (1975): Macht, Stuttgart.

Luhmann, Niklas (1990): Die Wissenschaft der Gesellschaft, Frankfurt / Main.

Luhmann, Niklas (2000): Organisation und Entscheidung, Opladen.

Mayntz, Renate (1985): Soziologie der öffentlichen Verwaltung, 3. Aufl., Heidelberg.

Meier, Frank (2009): Die Universität als Akteur. Zum institutionellen Wandel der Hochschulorganisation, Wiesbaden.

Meier, Frank / Uwe Schimank (2002): Szenarien der Profilbildung im deutschen Hochschulsystem. Einige Vermutungen, in: die hochschule 1, S. 82-91.

Mignot, Gerard (2003): Who Are the Actors in the Government of French Universities? The Paradoxal Victory of Deliberative Leadership, in: Higher Education 45, S. 71-89. 
Minssen, Heiner / Uwe Wilkesmann (2003): Lassen Hochschulen sich steuern? in: Soziale Welt 54, S. 123-141.

Mintzberg, Henry (1983): Power in and around Organizations, Englewood Cliffs.

Musselin, Christine (2005): European Academic Labor Markets in Transition, in: Higher Education 49, S. 135-154

Musselin, Christine (2010): The Markets for Academics, New York - London.

National Center for Education Statistics (NCES) Home Page, a part of the U.S. Department of Education, abrufbar unter http://nces.ed.gov, für die Zahlen zu 1980 / 81, vgl. http://nces.ed.gov/programs/digest/ d00/dt244.asp, für die Zahlen zu 2007 / 2008 vgl. http://nces.ed.gov/programs/digest/d09/tables/ dt09_264.asp, letztes Abrufdatum: 26.6.2011.

Neave, Guy / Gary Rhoades (1987): The Academic Estate in Western Europe, in: Burton Clark (Hrsg.), The Academic Profession. National, Disciplinary and Institutional Settings, Berkeley, S. 211-270.

Pollitt, Christopher / Sandra van Thiel / Vincent Homburg (Hrsg.) (2007): New Public Management in Europe, Basingstoke.

Rhoades, Gary / Barbara Sporn (2002): New Models of Management and Shifting Modes and Costs of Production: Europe and the United States, in: Tertiary Education and Management 8, S. 3-28.

Risser, Dominik (2003): Governance and Functioning of British Universities, in: Beiträge zur Hochschulforschung 25, S. 84-101.

Sahlin-Andersson, Kerstin (2002): National, International and Transnational Constructions of New Public Management, in: Tom Christensen / Per Lægreid (Hrsg.), New Public Management. The Transformation of Ideas and Practice, Aldershot, S. 43-72.

Scharpf, Fritz W. (1997): Games Real Actors Play, Boulder.

Scott, Richard W. (1968): Konflikte zwischen Spezialisten und bürokratischen Organisationen, in: Renate Mayntz (Hrsg.), Bürokratische Organisation, Köln, S. 201-216.

Seidler, Hans H. (2004): Hochschulfinanzierung, Evaluation und Mittelvergabe, in: Michael Hartmer / Hubert Detmer (Hrsg.), Hochschulrecht. Ein Handbuch für die Praxis, Heidelberg, S. 478-510.

Statistisches Bundesamt (2008): Bildung und Kultur: Personal an Hochschulen, Wiesbaden.

van Vught, Frans A. (1997): The Effects of Alternative Governance Structures. A Comparative Analysis of Higher Education Policy in Five EU Members State, in: Bernard Steunenberg / Frans A. van Vught (Hrsg.), Political Institutions and Public Policy. Perspectives on European Decision Making, Dordrecht, S. 115-137.

Vogel, Rick (2006): Zur Institutionalisierung von New Public Management. Disziplindynamik der Verwaltungswissenschaft unter dem Einfluss ökonomischer Theorie, Wiesbaden.

Weber, Max (1922): Wissenschaft als Beruf, in: Max Weber, Gesammelte Aufsätze zur Wissenschaftslehre, Tübingen, S. 524-555.

Weick, Karl E. (1976): Educational Organizations as Loosely Coupled Systems, in: Administrative Science Quarterly 21, S. 1-19.

Whitchurch, Celia (2006): Who Do They Think They Are? The Changing Identities of Professional Administrators and Managers in UK Higher Education, in: Journal of Higher Education Policy and Management 28, S. 159-171.

Wiesenthal, Helmut (2000): Markt, Organisation und Gemeinschaft als zweitbeste Verfahren sozialer Koordination, in: Raymund Werle / Uwe Schimank (Hrsg.), Gesellschaftliche Komplexität und kollektive Handlungsfähigkeit, Frankfurt / Main, S. 44-73.

Wissenschaftsrat (Hrsg.) (2007): Exzellenz in Wissenschaft und Forschung - Neue Wege in der Gleichstellungspolitik, Köln.

Zauner, Alfred (2005): Spannungsreiche Bewegungen. Gedanken zur Universität als Non-Profit Organisation, in: Heike Welte / Manfred Auer / Claudia Meister-Scheytt (Hrsg.), Management von Universitäten. Zwischen Tradition und (Post-)Moderne, München, S. 187-198. 
Dr. Otto Hüther Universität Hamburg Institut für Soziologie Allende-Platz 1 20146 Hamburg otto.huether@wiso.uni-hamburg.de

Prof. Dr. Georg Krücken Universität Kassel International Center for Higher Education Research (INCHER)

Mönchebergstr. 17 34109 Kassel kruecken@incher.uni-kassel.de 